\title{
EFEITOS DO USO DE ÓRTESE NA MOBILIDADE FUNCIONAL DE CRIANÇAS COM PARALISIA CEREBRAL
}

\author{
Cury VCR ${ }^{1}$, Mancini, MC ${ }^{2}$, Melo AP $^{1}$, Fonseca ST ${ }^{3}$, Sampaio RF $^{3}$, Tirado MGA ${ }^{2}$ \\ ${ }^{1}$ Escola de Educação Física, Fisioterapia e Terapia Ocupacional, Universidade Federal de Minas Gerais - UFMG, Belo \\ Horizonte, MG \\ 2 Departamento de Terapia Ocupacional, UFMG, Belo Horizonte, MG \\ ${ }^{3}$ Departamento de Fisioterapia, UFMG, Belo Horizonte, MG \\ Correspondência para: Valéria Cury, Rua: Tenente Anastácio Moura, 354, Apto. 301, Bairro Santa Efigênia, CEP: 30240- \\ 390, Belo Horizonte, MG, e-mail: valeriacury@hotmail.com
}

Recebido: 31/01/2005 - Aceito: 26/10/2005

\begin{abstract}
RESUMO
Objetivo: Comparar o desempenho motor de crianças com paralisia cerebral (PC) em duas condições: com órtese e sem órtese. Métodos: Vinte crianças PC foram avaliadas utilizando-se o teste Gross Motor Function Measure (GMFM), a versão modificada da avaliação da marcha Physicians Rating Scale (PRS) e entrevista com os pais para avaliar o uso de órteses na rotina diária. Resultados: O teste ANOVA que foi utilizado para avaliar o efeito do uso órtese na mobilidade das crianças revelou médias significantemente superiores na condição com órtese durante o desempenho motor grosso e na marcha. Entrevistas informaram que o uso de órteses estava inserido na rotina diária e os pais demonstraram percepção positiva com relação ao uso desse dispositivo. Conclusão: As órteses promoveram o desempenho de tarefas motoras da rotina diária de crianças com PC, podendo orientar os processos de avaliação e de intervenção dos profissionais que trabalham com essa clientela.
\end{abstract}

Palavras-chave: paralisia cerebral, órtese, mobilidade.

\section{ABSTRACT \\ The Effects of the Use of Orthoses on the Functional Mobility of Children with Cerebral Palsy}

Objective: To compare the motor performance of children with cerebral palsy (CP) under two conditions: with and without orthoses. Method: Twenty children with CP were evaluated using the Gross Motor Function Measure test (GMFM), a modified version of the Physicians Rating Scale (PRS) locomotion assessment, and an interview with their parents to evaluate the use of orthoses in their daily routine. Results: The ANOVA test was used to evaluate the effect of orthosis use on the children's mobility, and it revealed that, when orthosis was used, the means for gross motor function and gait assessments were significantly higher. The interviews confirmed that the use of orthoses was included in the daily routine, and the parents demonstrated positive perceptions regarding the use of such devices. Conclusion: Orthoses improved the performance of routine daily motor tasks among the children with $\mathrm{CP}$, and this finding may serve to guide the assessment and intervention processes of professionals who work with this clientele.

Key words: cerebral palsy, orthosis, mobility. 


\section{INTRODUÇÃO}

A paralisia cerebral (PC) é uma condição que, freqüentemente, interfere na aquisição de habilidades motoras na infância, as quais são essenciais para o desempenho de atividades e tarefas da rotina diária ${ }^{1,2,3,4}$. A locomoção é uma das atividades motoras que, geralmente, apresenta-se alterada em crianças com PC, quando comparadas com crianças normais.

Ao se considerar as capacidades motoras dessas crianças, as demandas da tarefa e as características do ambiente, observa-se que, muitas vezes, o padrão de locomoção apresentado por elas representa a melhor solução possível. Entretanto, algumas dessas soluções funcionais podem colocá-las em situação de risco para futuras alterações estruturais, como o aparecimento de encurtamentos, deformidades e prejuízo futuro na dinâmica da marcha ${ }^{5}$. Nesse contexto, as órteses de posicionamento são, freqüentemente, utilizadas para auxiliar no tratamento de crianças portadoras de paralisia cerebral. O suporte mecânico oferecido pelas órteses suropodálicas, por exemplo, visa minimizar ou corrigir o padrão eqüino de marcha assumido por algumas destas crianças, proporcionando benefícios como a melhora da qualidade de deambulação e a prevenção de deformidades de tornozelo e pé ${ }^{2,6}$.

Diversos autores documentaram os efeitos das órteses suropodálicas no padrão de locomoção de crianças com PC. De forma geral, foi observada diminuição da flexão plantar excessiva do tornozelo ${ }^{6}$, aumento do tamanho da passada ${ }^{6,7} \mathrm{e}$ maior estabilidade na fase de apoio ${ }^{8}$. Grande parte dos estudos informa sobre o impacto do uso dessas órteses nos componentes de desempenho neuromotor da criança. Poucos estudos descrevem o impacto das órteses suropodálicas durante a execução de atividades funcionais em contexto relevante da criança ${ }^{8,9}$. Entretanto, informações funcionais representam as principais queixas e expectativas da criança e de seus familiares ${ }^{10}$. Dessa forma, evidências centradas em componentes de desempenho, que não consideram diretamente a tarefa e o ambiente onde esses componentes se manifestam, fornecem um perfil incompleto da funcionalidade do indivíduo ${ }^{11}$.

A Classificação Internacional de Funcionalidade, Incapacidade e Saúde (CIF) foi publicada pela Organização Mundial de Saúde ${ }^{12}$ com o propósito de uniformizar a conceituação e terminologia dos processos de funcionalidade e incapacidade, bem como servir de parâmetro para organização de evidências. Segundo essa classificação, uma condição de saúde como a PC pode resultar em alterações da funcionalidade de uma criança que se manifestam em domínios de complexidades distintas. O domínio mais interno é denominado Estrutura e Função do Corpo e compreende os órgãos e sistemas, bem como a função dessas estruturas.
No caso da PC, as estruturas e funções neuromusculoesqueléticas apresentam-se comprometidas. Um outro domínio de funcionalidade definido pela CIF é denominado Atividade e Participação, que inclui o desempenho de atividades da rotina diária em contexto relevante, mas também o acesso e a participação ativa da criança na sociedade ${ }^{12}$. Nesse outro domínio, uma criança com PC pode apresentar limitações para desempenhar atividades típicas da infância como andar, subir e descer escadas, vestir-se e despir-se, além de encontrar barreiras que podem restringi-la de freqüentar escola com outras crianças ${ }^{13}$. Juntos, esses domínios incluem parâmetros internos e externos que influenciam os desfechos de funcionalidade e de incapacidade da criança.

Ao se utilizar a CIF $^{12}$ para organizar as evidências disponíveis na literatura, observa-se que grande parte dos estudos que investigaram o efeito de órteses em crianças portadoras de $\mathrm{PC}^{14}$ informa sobre o impacto desse dispositivo em variáveis que refletem o domínio de estrutura e função do corpo ${ }^{12}$. Informações sobre o impacto desse suporte no desempenho de atividades e tarefas típicas da rotina diária dessa clientela não têm sido disponibilizadas com a mesma freqüência ${ }^{8,15}$. Entretanto, tais informações são relevantes para profissionais que trabalham com esse grupo, podendo orientar procedimentos de avaliação e de intervenção, uma vez que a indicação para o uso de órteses visa a prevenção de deformidades e a facilitação de atividades motoras.

O objetivo deste estudo foi comparar a marcha e o desempenho motor grosso de crianças portadoras de PC em duas situações: sem órtese e com órtese. A avaliação da marcha teve como referência o padrão de marcha normal, e o desempenho motor das crianças foi avaliado em relação à capacidade das mesmas de realizar atividades da função motora grossa, tais como correr, pular e realizar transferências de posturas. Além disso, foram documentadas as percepções dos pais ou cuidadores sobre os efeitos do uso de órteses na rotina diária das crianças.

\section{METODOLOGIA}

\section{Participantes}

Vinte crianças com PC e seus pais ou responsáveis participaram desse estudo. Foram excluídas aquelas crianças que apresentavam distúrbios associados como retardo mental, malformações congênitas, síndromes genéticas, alterações sensoriais (i.e., visuais ou auditivas), bem como aquelas que foram submetidas a procedimentos cirúrgicos nos últimos 12 meses. Os critérios de inclusão para as crianças foram: capacidade de compreender ordens simples, gravidades leve e moderada do comprometimento motor (definido pela Classificação da Função Motora Grossa - GMFCS como níveis 1 a 3$)^{4}$, presença de eqüinismo durante a fase de apoio 
da marcha, uso regular de órteses por, pelo menos, 15 dias antes da coleta de dados. Durante a avaliação goniométrica, dever-se-ia observar a ausência de contratura em flexão do quadril com angulação superior a $20^{\circ}$, de flexão dos joelhos maior que $45^{\circ}$, observada na medida do ângulo poplíteo e amplitude do movimento de dorsoflexão do tornozelo, medida a partir do posicionamento da articulação subtalar em neutro, com restrição superior a $10^{\circ}$ de plantiflexão. Finalmente, as crianças deveriam receber prescrição de uso de órtese suropodálica, feita por médico ortopedista.

A gravidade da condição de paralisia cerebral foi definida utilizando-se a classificação da função motora grossa $(\mathrm{GMFCS})^{4}$. Segundo essa classificação, a função motora se baseia em informações sobre as limitações funcionais apresentadas por crianças com PC, necessidade de tecnologia assistiva e de dispositivos de suporte, tais como cadeiras de rodas e andadores. A gravidade da função motora é descrita em cinco níveis variando de 1 a 5, sendo: 1- limitações em atividades como correr e pular; 2 - limitações durante marcha em ambiente externo; 3- marcha com auxílio de dispositivos de suporte; 4- marcha com auxílio em ambiente interno; 5ausência de marcha. Nesse estudo, foram incluídas crianças com atividade motora grossa classificada nos níveis 1,2 e 3 dessa escala, que ilustram categorias leve e moderada de gravidade da PC.

As crianças deveriam estar inseridas em programa de reabilitação de uma clínica privada que conta com uma equipe multidisciplinar, e da Associação Mineira de Reabilitação (A.M.R.), ambas na cidade de Belo Horizonte, Minas Gerais. Além das crianças, esse estudo também contou com a participação de um de seus pais ou responsável.

\section{Instrumentação}

Esse estudo utilizou a seguinte instrumentação para coleta de dados: o teste Gross Motor Function Measure (GMFM) ${ }^{15}$ para quantificar o desempenho motor grosso (mobilidade, manutenção de posturas e transição) das crianças, a versão modificada da Physicians Rating Scale (PRS modificada) para sistematizar a documentação de parâmetros qualitativos da marcha ${ }^{17}$ e entrevista com um dos pais para avaliação de sua percepção sobre o impacto do uso de órteses na mobilidade de seu filho.

O GMFM é um teste padronizado desenvolvido para quantificar a função motora grossa de crianças portadoras de distúrbios neuromotores, particularmente, a paralisia cerebral $^{15}$.Esse teste inclui 88 itens que documentam atividades da função motora grossa em cinco dimensões ou sub-escalas: 1- deitando e rolando; 2- sentado; 3- engatinhando e ajoelhando; 4- de pé; 5- andando, correndo e pulando. As informações encontradas no manual do GMFM incluem critérios que especificam os escores para cada item ${ }^{16}$. Os itens em cada dimensão são compostos por atividades sensíveis às características motoras apresentadas por crianças portadoras de PC. Para esse estudo, foram administradas somente duas dimensões do teste GMFM: de pé, e andando/ correndo/pulando. Estudos que investigaram as propriedades psicométricas desse teste infantil informaram que o GMFM é um instrumento válido e confiável ${ }^{18,19}$.

A análise qualitativa da marcha foi realizada utilizandose a versão modificada da Physicians Rating Scale (PRS modificada). A PRS foi, inicialmente, desenvolvida por Koman et al. ${ }^{16}$ e, posteriormente, modificada por Boyd et al. ${ }^{17}$ para avaliar os resultados da aplicação da Toxina Botulínica tipo A na locomoção de indivíduos $\mathrm{PC}^{3,17}$ (Figura 1). A PRS modificada ${ }^{17}$ é uma escala observacional que sistematiza e quantifica a observação de componentes específicos da marcha. A criança pode receber pontuação máxima de 18 pontos que são distribuídos em 6 sub-escalas. Essa escala se baseia em parâmetros da marcha típica apresentados por crianças com desenvolvimento normal. A criança deve ser filmada em foco anteroposterior e lateral, enquanto deambula em uma distância média de 6 metros. A análise da marcha é realizada posteriormente, com a filmagem apresentada em câmera lenta, com base em critérios definidos pela escala (Figura 1).

A entrevista com um dos pais teve como objetivo avaliar se o uso de órteses estava realmente inserido no contexto da rotina diária da criança, além de considerar a opinião dos mesmos sobre os efeitos do uso deste dispositivo. O roteiro dessa entrevista foi desenvolvido especificamente para esse estudo, visando obtenção das seguintes informações: tempo médio de utilização da órtese em 24 horas; freqüência de utilização em ambientes interno (i.e., casa) e externo; impressão pessoal do cuidador sobre os efeitos da órtese na realização de atividades e tarefas de mobilidade, comparados com o desempenho motor sem o uso deste dispositivo; avaliação do cuidador sobre a aceitação da criança quanto à utilização da órtese.

\section{Procedimento}

A partir da prescrição ortopédica, foram confeccionadas órteses suropodálicas para todas as crianças participantes desse estudo. Crianças PC quadriplégicas e diplégicas espásticas usaram órteses bilaterais, as demais crianças PC hemiplégicas usaram órtese no membro inferior comprometido. Os modelos de órteses incluíram o tutor curto rígido, que mantém a articulação tibiotársica em postura rígida a $0^{\circ}$ de dorsoflexão e o tutor curto articulado, que permite o movimento de dorsoflexão e limita a flexão plantar da articulação tibiotársica, facilitando a progressão da tíbia sobre o pé, na fase de apoio da marcha. Considerados os prérequisitos para inclusão no estudo, os critérios para indicação de órtese rígida foram: limitação da amplitude normal do movimento de dorsoflexão do tornozelo, medida durante a avaliação goniométrica e necessidade de reforço muscular para maior estabilidade da pelve e tronco durante a postura 


\begin{tabular}{|c|c|c|c|c|c|}
\hline \multirow{2}{*}{\begin{tabular}{|c|} 
PARÂMETRO \\
1 - Posição do joelho em médio apoio
\end{tabular}} & \multirow[t]{2}{*}{ DEFINIÇÃO } & \multicolumn{2}{|c|}{$\begin{array}{c}\text { SEM } \\
\text { ÓRTESE }\end{array}$} & \multicolumn{2}{|c|}{$\begin{array}{c}\text { COM } \\
\text { ÓRTESE }\end{array}$} \\
\hline & & D & $\mathbf{E}$ & D & $\mathbf{E}$ \\
\hline & CROUCH - marcha agachada Genuflexão grave $>15^{\circ}$ & 0 & 0 & 0 & 0 \\
\hline & Genuflexão Moderada > $10-15^{\circ}$ & 1 & 1 & 1 & 1 \\
\hline & Genuflexão Leve $<10^{\circ}$ & 2 & 2 & 2 & 2 \\
\hline & Neutra & 3 & 3 & 3 & 3 \\
\hline & RECURVATO & 2 & 2 & 2 & 2 \\
\hline & Moderado $5-10^{\circ}$ & 1 & 1 & 1 & 1 \\
\hline & Grave $>10^{\circ}$ & 0 & 0 & 0 & 0 \\
\hline \multirow[t]{4}{*}{2 - Contato inicial do pé } & Dedo & 0 & 0 & 0 & 0 \\
\hline & Parte anterior do pé & 1 & 1 & 1 & 1 \\
\hline & Planta do pé & 2 & 2 & 2 & 2 \\
\hline & Calcanhar & 3 & 3 & 3 & 3 \\
\hline \multirow[t]{5}{*}{3 - Contato do pé no médio apoio } & Dedo/Dedo(equino) & -1 & -1 & -1 & -1 \\
\hline & Planta do pé / levantamento precoce do calcanhar & 0 & 0 & 0 & 0 \\
\hline & Planta do pé/ sem levantamento precoce do calcanhar & 1 & 1 & 1 & 1 \\
\hline & Calcanhar ocasional / planta do pé & 2 & 2 & 2 & 2 \\
\hline & Calcanhar / dedo (rolamento normal) & 3 & 3 & 3 & 3 \\
\hline \multirow[t]{5}{*}{4 - Tempo de levantamento do calcanhar } & Sem contato do calcanhar / equino fixo & 0 & 0 & 0 & 0 \\
\hline & Antes de $25 \%$ do médio apoio (muito precoce) & 1 & 1 & 1 & 1 \\
\hline & Entre 25 - 50\% (levantamento precoce) & 2 & 2 & 2 & 2 \\
\hline & No apoio terminal & 3 & 3 & 3 & 3 \\
\hline & Sem levant.do calcanhar (após apoio do pé, ex:crouch) & 0 & 0 & 0 & 0 \\
\hline \multirow[t]{4}{*}{5 - Base de suporte } & Caminhar em tesoura, clinicamente evidente & 0 & 0 & 0 & 0 \\
\hline & Base estreita (pouca liberação do joelho) & 1 & 1 & 1 & 1 \\
\hline & Base larga & 2 & 2 & 2 & 2 \\
\hline & Base normal, largura dos ombros & 3 & 3 & 3 & 3 \\
\hline \multirow[t]{4}{*}{6 - Dispositivos que auxiliam na marcha } & Andador (ant/post), com assistência & 0 & 0 & 0 & 0 \\
\hline & Andador (independente) & 1 & 1 & 1 & 1 \\
\hline & Muletas / Bengalas & 2 & 2 & 2 & 2 \\
\hline & Nenhum, independente por 10 metros & 3 & 3 & 3 & 3 \\
\hline
\end{tabular}

Score perfeito 18 por membro

Adaptado de: Boyd RN, Graham KH,1999.

Figura 1. Versão modificada da escala Physician’s Rating Scale (PRS modificado).

de pé. A indicação da órtese articulada levou em conta os seguintes critérios: ausência de restrições da amplitude de movimento de dorsoflexão do tornozelo, medida durante avaliação goniométrica ${ }^{20}$. Após a entrega dos dispositivos, as crianças tiveram um período de 15 dias para adaptação às novas órteses. Nesse período, foi verificada a presença ou não de áreas de hiperemia ou pontos de pressão, observados nas regiões envolvidas pela órtese, que pudessem justificar a necessidade de ajustes para facilitar sua utilização. Feitos os ajustes necessários, iniciou-se a contagem do tempo mínimo de 15 dias para uso em atividades da rotina diária. Os pais das crianças foram informados sobre os procedimentos do estudo e assinaram um termo de consentimento para a participação de seu filho. Esse estudo foi aprovado pelo Comitê de Ética em Pesquisa da Universidade Federal de Minas Gerais.

Todas as crianças participantes foram avaliadas por uma fisioterapeuta e uma terapeuta ocupacional com experiência na utilização dos instrumentos selecionados. Antes do início da coleta de dados, foi avaliada a confiabilidade entre as examinadoras obtendo-se índice de consistência superior a 0,80 .

O procedimento de coleta de dados teve início com a filmagem da marcha das crianças em local apropriado, para posterior análise através da escala PRS modificada. Os registros em filme foram feitos com a criança descalça e, em seguida, utilizando suas órteses e calçados habituais. Os membros inferiores de cada criança foram avaliados separadamente pelos parâmetros da PRS modificada. Em seguida, na aplicação do teste GMFM, as crianças foram testadas inicialmente descalças sem as órteses, e, posteriormente, a avaliação foi repetida com a criança usando órteses e calçados habituais, a fim de se obter informações para se proceder a comparação das duas situações. Por fim, foi feita entrevista com um dos pais da criança. 


\section{Análise Estatística}

Análises inferenciais incluíram análises de variância (ANOVA), que foram realizadas para cada uma das variáveis dependentes (escores do teste GMFM e escores da PRS). O primeiro teste ANOVA, com dois níveis de medidas repetidas comparou os efeitos condição (sem e com órtese), dimensão do teste GMFM (de pé; andando/correndo/pulando) e o fator de interação (condição x dimensão), nos escores obtidos pelas crianças no teste GMFM. O segundo teste ANOVA, que incluiu um nível da medida repetida e um fator independente, foi usado para avaliar os efeitos condição (sem e com órtese), diagnósticos de PC (quadriplegia, diplegia e hemiplegia), bem como o fator de interação (condição $x$ diagnóstico) na marcha das crianças (PRS modificada). Uma vez identificado efeito significativo nas análises descritas acima, contrastes préplanejados foram utilizados para localizar as diferenças (comparações post-hoc).

As análises inferenciais foram realizadas utilizando-se o pacote estatístico SUPERANOVA ${ }^{\circledR}$. Em todas as análises, foi considerado o nível de significância á =0,05.
A análise das entrevistas foi feita através da análise de conteúdo, destacando aqueles mais freqüentemente relatados pelos pais entrevistados, utilizando técnica de metodologia qualitativa.

\section{RESULTADOS}

A tabela 1 apresenta informação descritiva das crianças que compuseram os grupos 1 e 2, classificados de acordo com a gravidade da condição de PC.

Os resultados do primeiro teste ANOVA revelaram que somente os efeitos individuais órtese e dimensão apresentam significância estatística $\quad\left(\mathrm{F}_{\text {condição }}=37,253, \quad \mathrm{p}<0,0001\right.$; $\left.\mathrm{F}_{\text {dimensão }}=15,969, \mathrm{p}<0,0001\right)$. Contrastes pré-planejados indicaram que o desempenho das crianças com as órteses suropodálicas foi superior à condição sem órtese e que as médias dos escores obtidos no GMFM foram superiores na dimensão de pé, comparada com a dimensão andando/ correndo/pulando $(\mathrm{F}=37,958, \mathrm{p}<0,0001)$ (Tabela 2).

Tabela 1. Informação descritiva dos grupos de crianças PC nas variáveis idade, sexo e diagnóstico clínico.

\begin{tabular}{ccc}
\hline & \multicolumn{2}{c}{ Grupos } \\
\cline { 2 - 3 } Variáveis & $\mathbf{1}$ & $\mathbf{2}$ \\
& (Níveis 1,2 GMFCS) & (Nível 3 GMFCS) \\
\hline Idade* & $5,4(1,6)$ & $8,2(2,7)$ \\
Sexo** Feminino & 6 & 1 \\
Masculino & 7 & 6 \\
Diagnóstico clínico** Hemiplegia & 8 & - \\
Diplegia & 4 & 4 \\
Quadriplegia & 1 & 3 \\
\hline
\end{tabular}

* números indicam valores médios de idade em anos e desvio-padrão.

** números indicam freqüência $(\mathrm{N})$ de crianças portadoras de PC em cada categoria.

Tabela 2. Desempenho motor de crianças com paralisia cerebral nas duas dimensões do teste GMFM (de pé, e andando/correndo/pulando) e nos parâmetros qualitativos da marcha (escala PRS modificada), nas condições sem órtese e com órtese.

\begin{tabular}{lccc}
\hline Condição & GMFM Dimensão de & GMFM Dimensão & PRS modificada* \\
& pé* & A/C/P* & \\
\hline Sem órtese** & $59,51(31,3)$ & $51,07(29,8)$ & $6,95(3,9)$ \\
& {$[15,3-94,8]$} & {$[13,8-91,6]$} & {$[4-15]$} \\
Com órtese** & $62,07(31,4)$ & $53,07(29,7)$ & $10,91(3,4)$ \\
& {$[15,3-97,8]$} & {$[16,6-91,6]$} & {$[4-17]$} \\
\hline
\end{tabular}

* Diferença significativa entre condições $(\mathrm{p}<0,0001)$.

** Números indicam valores médios (desvio-padrão) e [mínimo - máximo]. 
Resultados do segundo teste ANOVA mostraram que o fator condição foi significativo ( $\mathrm{F}=27,927, \mathrm{p}<0,0001)$, mas nem o fator diagnóstico nem o fator de interação apresentaram significância estatística $\quad\left(\mathrm{F}_{\text {diagnóstico }}=2,043, \quad \mathrm{p}=0,1665\right.$; $\left.\mathrm{F}_{\text {diagnósticoX condição }}=0,239, \mathrm{p}=0,6297\right)$. Contrastes pré-planejados revelaram, mais uma vez, que crianças portadoras de PC apresentaram melhor desempenho nos aspectos qualitativos da marcha na condição com órtese, comparada com a condição sem órtese (Tabela 2).

O conteúdo das entrevistas realizadas com os pais revelou que o uso de órteses estava inserido na rotina diária das crianças participantes do estudo e que os benefícios desse dispositivo foram observados, principalmente, na locomoção em ambientes externos. Com relação ao tempo e a freqüência de utilização, os cuidadores relataram que "ele acorda, toma banho e põe o tutor. Só tira à noite”; “ele acorda, coloca o tutor e vai para escola. Chega e tira. Se tiver que sair, coloca de novo. Se não, fica sem o tutor”. A impressão pessoal dos pais sobre os efeitos da órtese na mobilidade funcional de seus filhos apontou para uma percepção de efeito predominantemente em atividades externas ao ambiente domiciliar, como ilustrado nas seguintes falas: "com o tutor, ele fica mais estável e mais firme para passear”; “quando está com o tutor, ele cai muito menos na rua do que quando não está”.

\section{DISCUSSÃO}

Os resultados deste estudo mostraram que o uso de órteses suropodálicas proporcionou benefícios tanto nos parâmetros qualitativos da marcha, quanto no desempenho motor grosso de crianças com PC. A observação mais detalhada de componentes qualitativos da marcha, através da PRS modificada, revelou que o uso das órteses suropodálicas influenciou, principalmente, o posicionamento do pé no contato inicial, assim como a progressão da distribuição de peso durante a fase de apoio, com redução do eqüinismo dinâmico observado neste grupo clínico. Esses achados confirmam os de outros estudos que utilizaram sistemas de análise de movimentos para verificar os benefícios do uso de órteses suropodálicas na marcha de crianças com PC $C^{7,21,22}$. A precisão da informação fornecida por esses sofisticados sistemas de análise de movimento é bastante superior à fornecida pela PRS modificada, entretanto, a utilização desses sistemas na avaliação clínica no Brasil ainda apresenta-se como uma realidade distante, devido ao alto custo de aquisição e de manutenção. Apesar de a PRS modificada ser uma escala observacional não padronizada e de não disponibilizar dados cinemáticos, esse instrumento mostrouse de fácil aplicação e capaz de detectar diferenças no padrão de marcha apresentado pelas crianças participantes do estudo, podendo ser considerado como um recurso adequado para a documentação de parâmetros qualitativos da marcha de crianças com PC, na rotina de serviços de reabilitação.

Outro resultado importante desse estudo refere-se ao benefício do uso de órteses no desempenho de atividades motoras como correr, e subir/descer escadas, que foram documentadas pelo GMFM. Esses achados sugerem que o uso de órteses pode facilitar o desempenho motor grosso observado durante as atividades motoras testadas no GMFM. Dois outros estudos recentes que utilizaram o teste GMFM investigaram os efeitos do uso de órteses suropodálicas em crianças com PC. Entretanto, não foram observadas, nesses estudos, diferenças significativas no desempenho motor grosso quando as crianças utilizavam as órteses prescritas ${ }^{23}$. Discrepâncias observadas entre os resultados dessas investigações e do presente estudo podem estar relacionadas às características dos participantes tais como idade e gravidade da condição de PC.

Maltais et al. ${ }^{22}$ compararam o desempenho de dez crianças portadoras de diplegia com idade média de 9 anos, nas condições com e sem órtese, utilizando os itens das dimensões de pé e andando/correndo/pulando do teste GMFM. Nove crianças participantes desse estudo apresentavam grau leve de gravidade da condição de PC (níveis 1 e 2 da classificação GMFCS). Os resultados revelaram que o uso de órtese não influenciou significativamente os escores do teste GMFM. Os autores argumentaram que a ausência de diferença significativa poderia estar relacionada à baixa sensibilidade do teste em detectar mudanças na função motora grossa associada ao uso de órtese. Entretanto, o GMFM apresenta menor sensibilidade para detectar mudanças em crianças com idades superiores a seis anos e inferiores a três anos e, também, naquelas que apresentam escores nos limites superiores ou inferiores do mesmo (categorias extremas de comprometimento motor) ${ }^{19}$. A justificativa para os resultados não significativos encontrados por Maltais et al..$^{22}$ pode estar relacionada às características da amostra utilizada, incluindo crianças com média de idade superior a seis anos e gravidade leve da condição de PC (níveis 1 e 2 da GMFCS). Tais características ilustram, provavelmente, o efeito teto do teste, conforme ressaltado pelos autores do GMFM $^{18}$. Em nosso estudo, a amostra selecionada incluiu crianças portadoras de PC com média de idade mais baixa (6,4 anos) e que apresentaram maior diversidade de comprometimento da função motora grossa (níveis 1, 2 e 3 da GMFCS). É possível que a significância estatística encontrada em nossos resultados na condição com órtese comparada com a condição sem órtese possa ser atribuída a essas características indicadas acima. Essa mesma argumentação (idade e gravidade da condição de PC) pode justificar os resultados apresentados pelo estudo de Hassani et al. ${ }^{23}$, no qual também não foram documentadas diferenças significativas na atividade motora grossa de crianças com PC, na condição com órtese comparada com a condição sem 
órtese. Essa argumentação pode limitar a validade externa dos estudos que investigaram o efeito do uso de órtese na mobilidade de crianças com PC, dificultando a generalização dos resultados para crianças cujas características não estejam representadas no grupo amostral.

Durante a avaliação da marcha das crianças com órtese, não foram observadas diferenças significativas na comparação dos grupos por diagnóstico. Esse resultado indica que o uso de órteses favoreceu a qualidade da marcha de crianças com PC, independente do diagnóstico topográfico apresentado.

As narrativas dos pais durante a entrevista ressaltaram, predominantemente, aspectos favoráveis do uso de órteses, os quais não foram quantificados pela aplicação dos instrumentos de avaliação utilizados neste estudo. Essas observações revelaram a percepção positiva dos pais com relação ao uso de órteses suropodálicas na qualidade da locomoção, assim como no desempenho de atividades da rotina diária dessas crianças.

De acordo a CIF, os achados desse estudo indicaram benefícios com a utilização das órteses suropodálicas observados no domínio da Atividade e Participação, pela melhora do desempenho de atividades típicas da infância como correr, abaixar, durante as transferências de posturas e na locomoção em ambiente externo, observados pela utilização do GMFM e da entrevista com os cuidadores. Tal efeito funcional pode ser resultado de uma maior estabilidade do pé devido ao maior grau de dorsoflexão na fase de contato inicial da marcha dessas crianças (posição de ajuste máximo da articulação) e posicionamento plantígrado durante a fase de apoio da marcha, observados pela utilização da PRS, que ilustram os efeitos também no domínio da Estrutura e Função do Corpo.

Os resultados desse estudo reforçam a indicação das órteses suropodálicas como auxiliares a outras técnicas de tratamento da criança PC, que têm como objetivo melhorar a qualidade da locomoção e facilitar a mobilidade funcional.

Agradecimentos: à Associação Mineira de Reabilitação (AMR) e à clínica privada que colaboraram diretamente com o estudo e, também, aos pais e cuidadores das crianças.

\section{REFERÊNCIAS BIBLIOGRÁFICAS}

1. Suputtitada A. Managing spasticity in pediatric cerebral palsy using a very low dose of botulinum toxin type A. Am J Phys Med Rehabil 2000; 79: 320-26.

2. Burtner PA, Woollacott MH, Qualls C. Stance balance control with orthoses in a group of children with spastic cerebral palsy. Dev Med Child Neurol 1999; 41: 748-57.
3. Kooman LA, Mooney JF, Smith BP, Walker F, Leon JM. Botulinum toxin type A neuromuscular blockade in the treatment of lower extremity spasticity in cerebral palsy: a randomized, double-blind, placebo-controlled trial. J Pediatr Orthop 2000; 20: $108-15$

4. Palisano R, Rosenbaum P, Walters S, Russeal DJ, Wood EP, Galupp P. Development and reliability of a system to classify gross motor function in children with cerebral palsy. Dev Med Child Neurol 1997; 39: 214-23.

5. Cusick B. An Overview of components and concepts involved in orthotic prescription for children with cerebral palsy. In: Report of a Consensus Conference on the Lower Limb Orthotic Management of Cerebral Palsy. Durhan, N Carolina: International Society for Prostetic and Orthotics; 1994. 94-122.

6. Radtka SA, Skinner SR, Dixon DM, Johanson ME. A comparison of gait with solid, dynamic, and no ankle-foot orthoses in children with spastic cerebral palsy. Phys Ther 1997; 77: 395-409.

7. Rethlefsen S, Kay R, Dennis S, Forstein M, Tolo V. The effects of fixed and articulated ankle-foot orthoses on gait patterns in subjects with cerebral palsy. J Pediatr Orthop 1999; 19: 470-74.

8. Wilson H, Haideri N, Song K, Telford D. Ankle-foot orthoses for preambulatory children with spastic diplegia. J Pediatr Orthop 1997; 17: 370-76.

9. Morris C. A review of the efficacy of lower-limb orthoses used for cerebral palsy. Dev Med Child Neurol 2002; 44: 205-11.

10. Keith RA. Conceptual basis of outcome measures. Am J Phys Med Rehabil 1995; 74: 73-80.

11. Haley SM, Coster WC, Binda-Sundberg K. Measuring physical disablement: the contextual challenge. Phys Ther 1994; 74: 44351.

12. World Health Organization. International Classification of Functioning, Disability and Health (ICF). Geneva: World Health Organization; 2001.

13. Buckon CE, Thomas SS, Huston SJ, Aiona MD, Sussman MD. A preliminary comparison of the effect of solid, hinged and posterior leaf spring AFOs on the gait of children with spastic hemiplegia and diplegia. Gait Posture 1999; 9: 105.

14. Haideri N, Song K, Wilson H, Telford CO. The effects of solid and articulating ankle foot orthosis during sit-to-stand in young children with spastic diplegia. Gait Posture 1995; 3: 98.

15. Russell D, Rosembaum P, Gowland C et al. Gross Motor Function Measure (GMFM-66 \& GMFM-88) User's Manual. Ed. Hamilton, Ontario, Canada: Mac Keith Press; 2002.

16. Koman AL, Mooney JF, Smith BP, Goodman A. Management of cerebral palsy with botulinum toxin A: preliminary investigation. J Pediatr Orthop 1993; 13: 489-95.

17. Boyd RN, Graham KH. Objective measurement of clinical findings in the use of botulinum toxin type A for the management of children with cerebral palsy. Eur J Neurol 1999; 6: 23-35.

18. Russell DJ, Avery LM, Rosembaum P, Raina PS, Walter SD, Palisano RJ. Improved scaling of the gross motor function measure for children with cerebral palsy: evidence of reliability and validity. Phys Ther 2000; 80: 873-85. 
19. Koman LA, Mooney JF, Smith BP, Goodman A. Management of cerebral palsy with botulinum toxin A: a report of preliminary randomized, double-blind trial. J Pediatr Orthop 1994; 14: 229-303.

20. White H, Jenkins J, Neace WP, Tylkowski C, Walker J. Clinically prescribed orthosis demonstrate increase in velocity of gait in children with cerebral palsy: a retrospective study. Dev Med Child Neurol 2002; 44: 227-32.

21. Dursun E, Dursun N, Alican D. Ankle-foot orthoses: effect on gait in children with cerebral palsy. Disabil Rehabil 2002; 34547.

22. Maltais D, Bar-or O, Galea V, Pierrynowski M. Use of orthoses lowers the $\mathrm{O} 2$ cost of walking in children with spastic cerebral palsy. Med Sci Sports Exerc 2001; 33: 320-25.

23. Hassani S, Ferdjallah M, Reiners K, Johnson C, Smith P, Harris G. Motor performance comparison of the hinged and dynamic ankle foot orthotics. Abstracts. AACPDM, 2002. p.44: 34. 